\title{
Oscillation Behavior of Third-Order Neutral Emden-Fowler Delay Dynamic Equations on Time Scales
}

\author{
Zhenlai Han, ${ }^{1,2}$ Tongxing Li, ${ }^{1}$ Shurong Sun, ${ }^{1,3}$ \\ and Chenghui Zhang ${ }^{2}$ \\ ${ }^{1}$ School of Science, University of Jinan, Jinan, Shandong 250022, China \\ ${ }^{2}$ School of Control Science and Engineering, Shandong University, Jinan, Shandong 250061, China \\ ${ }^{3}$ Department of Mathematics and Statistics, Missouri University of Science and Technology, \\ Rolla, MO 65409-0020, USA
}

Correspondence should be addressed to Shurong Sun, sshrong@163.com

Received 14 September 2009; Revised 28 November 2009; Accepted 10 December 2009

Academic Editor: Leonid Berezansky

Copyright (C) 2010 Zhenlai Han et al. This is an open access article distributed under the Creative Commons Attribution License, which permits unrestricted use, distribution, and reproduction in any medium, provided the original work is properly cited.

\begin{abstract}
We will establish some oscillation criteria for the third-order Emden-Fowler neutral delay dynamic equations $\left(r(t)(x(t)-a(t) x(\tau(t)))^{\Delta \Delta}\right)^{\Delta}+p(t) x^{\gamma}(\delta(t))=0$ on a time scale $\mathbb{T}$, where $\gamma>0$ is a quotient of odd positive integers with $r, a$, and $p$ real-valued positive rd-continuous functions defined on $\mathbb{T}$. To the best of our knowledge nothing is known regarding the qualitative behavior of these equations on time scales, so this paper initiates the study. Some examples are considered to illustrate the main results.
\end{abstract}

\section{Introduction}

The study of dynamic equations on time-scales, which goes back to its founder Hilger [1], is an area of mathematics that has recently received a lot of attention. It has been created in order to unify the study of differential and difference equations. Many results concerning differential equations carry over quite easily to corresponding results for difference equations, while other results seem to be completely different from their continuous counterparts. The study of dynamic equations on time-scales reveals such discrepancies, and helps avoid proving results twice-once for differential equations and once again for difference equations.

Several authors have expounded on various aspects of this new theory; see the survey paper by Agarwal et al. [2], Bohner and Guseinov [3], and references cited therein. A book 
on the subject of time-scales, by Bohner and Peterson [4], summarizes and organizes much of the time-scale calculus; see also the book by Bohner and Peterson [5] for advances in dynamic equations on time-scales.

In the recent years, there has been increasing interest in obtaining sufficient conditions for the oscillation and nonoscillation of solutions of various equations on time-scales; we refer the reader to the papers [6-38]. To the best of our knowledge, it seems to have few oscillation results for the oscillation of third-order dynamic equations; see, for example, [14$16,21,35]$. However, the paper which deals with the third-order delay dynamic equation is due to Hassan [21].

Hassan [21] considered the third-order nonlinear delay dynamic equations

$$
\left(c(t)\left(\left(a(t) x^{\Delta}(t)\right)^{\Delta}\right)^{\gamma}\right)^{\Delta}+f(t, x(\tau(t)))=0, \quad t \in \mathbb{T}
$$

where $\tau(\sigma(t))=\sigma(\tau(t))$ is required, and the author established some oscillation criteria for (1.1) which extended the results given in [16].

To the best of our knowledge, there are no results regarding the oscillation of the solutions of the following third-order nonlinear neutral delay dynamic equations on timescales up to now:

$$
\left(r(t)(x(t)-a(t) x(\tau(t)))^{\Delta \Delta}\right)^{\Delta}+p(t) x^{\gamma}(\delta(t))=0, \quad t \in \mathbb{T} .
$$

We assume that $\gamma>0$ is a quotient of odd positive integers, $r, a$ and $p$ are positive real-valued rd-continuous functions defined on $\mathbb{T}$ such that $r^{\Delta}(t) \geq 0,0<a(t) \leq a_{0}<$ $1, \lim _{t \rightarrow \infty} a(t)=a<1$, the delay functions $\tau: \mathbb{T} \rightarrow \mathbb{T}, \delta: \mathbb{T} \rightarrow \mathbb{T}$ are rd-continuous functions such that $\tau(t) \leq t, \delta(t) \leq t$, and $\lim _{t \rightarrow \infty} \tau(t)=\lim _{t \rightarrow \infty} \delta(t)=\infty$.

As we are interested in oscillatory behavior, we assume throughout this paper that the given time-scale $\mathbb{T}$ is unbounded above. We assume $t_{0} \in \mathbb{T}$ and it is convenient to assume $t_{0}>0$. We define the time-scale interval of the form $\left[t_{0}, \infty\right)_{\mathbb{T}}$ by $\left[t_{0}, \infty\right)_{\mathbb{T}}=\left[t_{0}, \infty\right) \cap \mathbb{T}$.

For the oscillation of neutral delay dynamic equations on time-scales, Mathsen et al. [26] considered the first-order neutral delay dynamic equations on time-scales

$$
[y(t)-r(t) y(\tau(t))]^{\Delta}+p(t) y(\delta(t))=0, \quad t \in \mathbb{T},
$$

and established some new oscillation criteria of (1.3) which as a special case involve some well-known oscillation results for first-order neutral delay differential equations.

Agarwal et al. [7], Şahíner [28], Saker [31], Saker et al. [33], Wu et al. [34] studied the second-order nonlinear neutral delay dynamic equations on time-scales

$$
\left(r(t)\left((y(t)+p(t) y(\tau(t)))^{\Delta}\right)^{r}\right)^{\Delta}+f(t, y(\delta(t)))=0, \quad t \in \mathbb{T},
$$

by means of Riccati transformation technique, the authors established some oscillation criteria of (1.4). 
Saker [32] investigated the second-order neutral Emden-Fowler delay dynamic equations on time-scales

$$
\left[a(t)(y(t)+r(t) y(\tau(t)))^{\Delta}\right]^{\Delta}+p(t) y^{\gamma}(\delta(t))=0, \quad t \in \mathbb{T},
$$

and established some new oscillation for (1.5).

Our purpose in this paper is motivated by the question posed in [26]: What can be said about higher-order neutral dynamic equations on time-scales and the various generalizations? We refer the reader to the articles $[23,24]$ and we will consider the particular case when the order is 3 , that is, (1.2). Set $t_{-1}:=\min _{t \in\left[t_{0}, \infty\right)_{\mathbb{T}}}\{\tau(t), \delta(t)\}$. By a solution of (1.2), we mean a nontrivial real-valued function $x \in C_{r d}\left(\left[t_{-1}, \infty\right)_{\mathbb{T}}, \mathbb{R}\right)$ satisfying $x-a x \circ \tau \in$ $C_{r d}^{2}\left(\left[t_{0}, \infty\right)_{\mathbb{T}}, \mathbb{R}\right)$ and $r(x-a x \circ \tau)^{\Delta \Delta} \in C_{r d}^{1}\left(\left[t_{0}, \infty\right)_{\mathbb{T}}, \mathbb{R}\right)$, and satisfying $(1.2)$ for all $t \in\left[t_{0}, \infty\right)_{\mathbb{T}}$.

The paper is organized as follows. In Section 2, we apply a simple consequence of Keller's chain rule, devoted to the proof of the sufficient conditions which guarantee that every solution of (1.2) oscillates or converges to zero. In Section 3, some examples are considered to illustrate the main results.

\section{Main Results}

In this section we give some new oscillation criteria for (1.2). In order to prove our main results, we will use the formula

$$
\left((x(t))^{\gamma}\right)^{\Delta}=\gamma \int_{0}^{1}\left[h x^{\sigma}(t)+(1-h) x(t)\right]^{\gamma-1} x^{\Delta}(t) \mathrm{d} h,
$$

where $x$ is delta differentiable and eventually positive or eventually negative, which is a simple consequence of Keller's chain rule (see Bohner and Peterson [4, Theorem 1.90]).

Before stating our main results, we begin with the following lemmas which are crucial in the proofs of the main results.

For the sake of convenience, we denote: $z(t)=x(t)-a(t) x(\tau(t))$, for $t \in\left[t_{0}, \infty\right)_{\mathbb{T}}$. Also, we assume that

$(H)$ there exists $\left\{c_{k}\right\}_{k \in \mathbb{N}_{0}} \subset \mathbb{T}$ such that $\lim _{k \rightarrow \infty} c_{k}=\infty$ and $\tau\left(c_{k+1}\right)=c_{k}$.

Lemma 2.1. Assume that $(H)$ holds. Further, assume that $x$ is an eventually positive solution of (1.2). If

$$
\int_{t_{0}}^{\infty} \frac{\Delta t}{r(t)}=\infty
$$

then there are only the following three cases for $t \geq t_{1}$ sufficiently large:

(i) $z(t)>0, z^{\Delta}(t)>0, z^{\Delta \Delta}(t)>0, z^{\Delta \Delta \Delta}(t)<0$,

(ii) $z(t)<0, z^{\Delta}(t)>0, z^{\Delta \Delta}(t)>0, z^{\Delta \Delta \Delta}(t)<0, \lim _{t \rightarrow \infty} x(t)=0$,

or

(iii) $z(t)>0, z^{\Delta}(t)<0, z^{\Delta \Delta}(t)>0, z^{\Delta \Delta \Delta}(t)<0, \lim _{t \rightarrow \infty} z(t)=l \geq 0, \lim _{t \rightarrow \infty} x(t)=$ $l /(1-a) \geq 0$. 
Proof. Let $x$ be an eventually positive solution of (1.2). Then there exists $t_{1} \geq t_{0}$ such that $x(t)>0, x(\tau(t))>0$, and $x(\delta(t))>0$ for all $t \geq t_{1}$. From (1.2) we have

$$
\left(r(t) z^{\Delta \Delta}(t)\right)^{\Delta}=-p(t) x^{\gamma}(\delta(t))<0, \quad t \geq t_{1}
$$

Hence $r(t) z^{\Delta \Delta}(t)$ is strictly decreasing on $\left[t_{1}, \infty\right)_{\mathbb{T}}$. We claim that $z^{\Delta \Delta}(t)>0$ eventually. Assume not, then there exists $t_{2} \geq t_{1}$ such that

$$
r(t) z^{\Delta \Delta}(t)<0, \quad t \geq t_{2}
$$

Then we can choose a negative $c$ and $t_{3} \geq t_{2}$ such that

$$
r(t) z^{\Delta \Delta}(t) \leq c<0, \quad t \geq t_{3}
$$

Dividing by $r(t)$ and integrating from $t_{3}$ to $t$, we have

$$
z^{\Delta}(t) \leq z^{\Delta}\left(t_{3}\right)+c \int_{t_{3}}^{t} \frac{\Delta s}{r(s)} .
$$

Letting $t \rightarrow \infty$, then $z^{\Delta}(t) \rightarrow-\infty$ by (2.2). Thus, there is a $t_{4} \geq t_{3}$ such that for $t \geq t_{4}$

$$
z^{\Delta}(t) \leq z^{\Delta}\left(t_{4}\right)<0
$$

Integrating the previous inequality from $t_{4}$ to $t$, we obtain

$$
z(t)-z\left(t_{4}\right) \leq z^{\Delta}\left(t_{4}\right)\left(t-t_{4}\right)
$$

Therefore, there exist $d>0$ and $t_{5} \geq t_{4}$ such that

$$
x(t) \leq-d+a(t) x(\tau(t)) \leq-d+a_{0} x(\tau(t)), \quad t \geq t_{5} .
$$

We can choose some positive integer $k_{0}$ such that $c_{k} \geq t_{5}$, for $k \geq k_{0}$. Thus, we obtain

$$
\begin{aligned}
x\left(c_{k}\right) & \leq-d+a_{0} x\left(\tau\left(c_{k}\right)\right)=-d+a_{0} x\left(c_{k-1}\right) \leq-d-a_{0} d+a_{0}^{2} x\left(\tau\left(c_{k-1}\right)\right) \\
& =-d-a_{0} d+a_{0}^{2} x\left(c_{k-2}\right) \leq \cdots \leq-d-a_{0} d-\cdots-a_{0}^{k-k_{0}-1} d+a_{0}^{k-k_{0}} x\left(\tau\left(c_{k_{0}+1}\right)\right) \\
& =-d-a_{0} d-\cdots-a_{0}^{k-k_{0}-1} d+a_{0}^{k-k_{0}} x\left(c_{k_{0}}\right) .
\end{aligned}
$$

The above inequality implies that $x\left(c_{k}\right)<0$ for sufficiently large $k$, which contradicts the fact that $x(t)>0$ eventually. Hence we get

$$
z^{\Delta \Delta}(t)>0
$$


It follows from this that either $z^{\Delta}(t)>0$ or $z^{\Delta}(t)<0$. Since $r^{\Delta}(t) \geq 0$,

$$
\left(r(t) z^{\Delta \Delta}(t)\right)^{\Delta}=r^{\Delta}(t) z^{\Delta \Delta}(t)+r^{\sigma}(t) z^{\Delta \Delta \Delta}(t)<0,
$$

which yields

$$
z^{\Delta \Delta \Delta}(t)<0
$$

If $z^{\Delta}(t)>0$, then there are two possible cases:

(1) $z(t)>0$, eventually; or

(2) $z(t)<0$, eventually.

If there exists a $t_{6} \geq t_{1}$ such that case (2) holds, then $\lim _{t \rightarrow \infty} z(t)$ exists, and $\lim _{t \rightarrow \infty} z(t)=b \leq 0$. We claim that $\lim _{t \rightarrow \infty} z(t)=0$. Otherwise, $\lim _{t \rightarrow \infty} z(t)=b<0$. We can choose some positive integer $k_{0}$ such that $c_{k} \geq t_{6}$, for $k \geq k_{0}$. Thus, we obtain

$$
\begin{aligned}
x\left(c_{k}\right) & \leq a_{0} x\left(\tau\left(c_{k}\right)\right)=a_{0} x\left(c_{k-1}\right) \leq a_{0}^{2} x\left(\tau\left(c_{k-1}\right)\right) \\
& =a_{0}^{2} x\left(c_{k-2}\right) \leq \cdots \leq a_{0}^{k-k_{0}} x\left(\tau\left(c_{k_{0}+1}\right)\right)=a_{0}^{k-k_{0}} x\left(c_{k_{0}}\right),
\end{aligned}
$$

which implies that $\lim _{k \rightarrow \infty} x\left(c_{k}\right)=0$, and from the definition of $z(t)$, we have $\lim _{k \rightarrow \infty} z\left(c_{k}\right)=$ 0 , which contradicts $\lim _{t \rightarrow \infty} z(t)<0$. Now, we assert that $x$ is bounded. If it is not true, there exists $\left\{s_{k}\right\}_{k \in \mathbb{N}} \subset\left[t_{6}, \infty\right)_{\mathbb{T}}$ with $s_{k} \rightarrow \infty$ as $k \rightarrow \infty$ such that

$$
x\left(s_{k}\right)=\sup _{t_{0} \leq s \leq s_{k}} x(s), \quad \lim _{k \rightarrow \infty} x\left(s_{k}\right)=\infty .
$$

From $\tau(t) \leq t$

$$
z\left(s_{k}\right)=x\left(s_{k}\right)-a\left(s_{k}\right) x\left(\tau\left(s_{k}\right)\right) \geq\left(1-a_{0}\right) x\left(s_{k}\right),
$$

which implies that $\lim _{k \rightarrow \infty} z\left(s_{k}\right)=\infty$, it contradicts that $\lim _{t \rightarrow \infty} z(t)=0$. Therefore, we can assume that

$$
\limsup _{t \rightarrow \infty} x(t)=x_{1}, \quad \liminf _{t \rightarrow \infty} x(t)=x_{2} .
$$

By $0 \leq a<1$, we get

$$
x_{1}-a x_{1} \leq 0 \leq x_{2}-a x_{2}
$$

which implies that $x_{1} \leq x_{2}$, so $x_{1}=x_{2}$, hence, $\lim _{t \rightarrow \infty} x(t)=0$. 
Assume that $z^{\Delta}(t)<0$. We claim that $z(t) \geq 0$ eventually. Otherwise, we have $\lim _{t \rightarrow \infty} z(t)<0$ or $\lim _{t \rightarrow \infty} z(t)=-\infty$. By $(H)$, there exists $t_{7} \geq t_{1}$, we can choose some positive integer $k_{0}$ such that $c_{k} \geq t_{7}$ for $k \geq k_{0}$, and we obtain

$$
\begin{aligned}
x\left(c_{k}\right) & \leq a_{0} x\left(\tau\left(c_{k}\right)\right)=a_{0} x\left(c_{k-1}\right) \leq a_{0}^{2} x\left(\tau\left(c_{k-1}\right)\right) \\
& =a_{0}^{2} x\left(c_{k-2}\right) \leq \cdots \leq a_{0}^{k-k_{0}} x\left(\tau\left(c_{k_{0}+1}\right)\right)=a_{0}^{k-k_{0}} x\left(c_{k_{0}}\right),
\end{aligned}
$$

which implies that $\lim _{k \rightarrow \infty} x\left(c_{k}\right)=0$, and from the definition of $z$, we have $\lim _{k \rightarrow \infty} z\left(c_{k}\right)=0$, which contradicts $\lim _{t \rightarrow \infty} z(t)<0$ or $\lim _{t \rightarrow \infty} z(t)=-\infty$. Now, we have that $\lim _{t \rightarrow \infty} z(t)=l \geq 0$, here $l$ is finite. We assert that $x$ is bounded. If it is not true, there exists $\left\{s_{k}\right\}_{k \in \mathbb{N}} \subset\left[t_{6}, \infty\right)_{\mathbb{T}}$ with $s_{k} \rightarrow \infty$ as $k \rightarrow \infty$ such that

$$
x\left(s_{k}\right)=\sup _{t_{0} \leq s \leq s_{k}} x(s), \quad \lim _{k \rightarrow \infty} x\left(s_{k}\right)=\infty .
$$

From $\tau(t) \leq t$

$$
z\left(s_{k}\right)=x\left(s_{k}\right)-a\left(s_{k}\right) x\left(\tau\left(s_{k}\right)\right) \geq\left(1-a_{0}\right) x\left(s_{k}\right),
$$

which implies that $\lim _{k \rightarrow \infty} z\left(s_{k}\right)=\infty$, it contradicts that $\lim _{t \rightarrow \infty} z(t)=l \geq 0$. Therefore, we can assume that

$$
\limsup _{t \rightarrow \infty} x(t)=x_{1 *,} \quad \liminf _{t \rightarrow \infty} x(t)=x_{2 *}
$$

By $0 \leq a<1$, we get

$$
x_{1 *}-a x_{1 *} \leq l \leq x_{2 *}-a x_{2 *}
$$

which implies that $x_{1 *} \leq x_{2 *}$, so $x_{1 *}=x_{2 *}$, hence, $\lim _{t \rightarrow \infty} x(t)=l /(1-a) \geq 0$. This completes the proof.

In $\left[4\right.$, Section 1.6] the Taylor monomials $\left\{h_{n}(t, s)\right\}_{n=0}^{\infty}$ are defined recursively by

$$
h_{0}(t, s)=1, \quad h_{n+1}(t, s)=\int_{s}^{t} h_{n}(\tau, s) \Delta \tau, \quad t, s \in \mathbb{T}, n \geq 1 .
$$

It follows from [4, Section 1.6] that $h_{1}(t, s)=t-s$ for any time-scale, but simple formulas in general do not hold for $n \geq 2$. 
Lemma 2.2 (see [15, Lemma 4]). Assume that $z$ satisfies case (i) of Lemma 2.1. Then

$$
\liminf _{t \rightarrow \infty} \frac{t z(t)}{h_{2}\left(t, t_{0}\right) z^{\Delta}(t)} \geq 1
$$

Lemma 2.3. Assume that $x$ is a solution of (1.2) satisfying case (i) of Lemma 2.1. If

$$
\int_{t_{0}}^{\infty} p(t)\left(h_{2}\left(\delta(t), t_{0}\right)\right)^{\gamma} \Delta t=\infty
$$

then $z$ satisfies eventually

$$
z^{\Delta}(t) \geq t z^{\Delta \Delta}(t), \quad \frac{z^{\Delta}(t)}{t} \text { is nonincreasing. }
$$

Proof. Let $x$ be a solution of (1.2) such that case (i) of Lemma 2.1 holds for $t \geq t_{1}$. Define

$$
Z(t)=z^{\Delta}(t)-t z^{\Delta \Delta}(t)
$$

Thus

$$
Z^{\Delta}(t)=-\sigma(t) z^{\Delta \Delta \Delta}(t)>0
$$

We claim that $Z(t)>0$ eventually. Otherwise, there exists $t_{2} \geq t_{1}$ such that $Z(t)<0$ for $t \geq t_{2}$. Therefore,

$$
\left(\frac{z^{\Delta}(t)}{t}\right)^{\Delta}=-\frac{Z(t)}{t \sigma(t)}>0, \quad t \geq t_{2}
$$

which implies that $z^{\Delta}(t) / t$ is strictly increasing on $\left[t_{2}, \infty\right)_{\mathbb{T}}$. Pick $t_{3} \geq t_{2}$ such that $\delta(t) \geq \delta\left(t_{3}\right) \geq$ $t_{2}$, for $t \geq t_{3}$. Then we have

$$
\frac{z^{\Delta}(\delta(t))}{\delta(t)} \geq \frac{z^{\Delta}\left(\delta\left(t_{3}\right)\right)}{\delta\left(t_{3}\right)}=P>0
$$

then $z^{\Delta}(\delta(t)) \geq P \delta(t)$ for $t \geq t_{3}$. By Lemma 2.2, for any $0<k<1$, there exists $t_{4} \geq t_{3}$ such that

$$
\frac{z(t)}{z^{\Delta}(t)} \geq k \frac{h_{2}\left(t, t_{0}\right)}{t}, \quad t \geq t_{4}
$$

Hence there exists $t_{5} \geq t_{4}$ so that

$$
z(\delta(t)) \geq k \frac{h_{2}\left(\delta(t), t_{0}\right)}{\delta(t)} z^{\Delta}(\delta(t)) \geq P k \frac{h_{2}\left(\delta(t), t_{0}\right)}{\delta(t)} \delta(t)=P k h_{2}\left(\delta(t), t_{0}\right), \quad t \geq t_{5}
$$


By the definition of $z$, we have that

$$
x(t) \geq z(t)
$$

From (1.2), we obtain

$$
\left(r(t) z^{\Delta \Delta}(t)\right)^{\Delta}+p(t) z^{\gamma}(\delta(t)) \leq 0
$$

Integrating both sides of (2.35) from $t_{5}$ to $t$, we get

$$
r(t) z^{\Delta \Delta}(t)-r\left(t_{5}\right) z^{\Delta \Delta}\left(t_{5}\right)+(P k)^{r} \int_{t_{5}}^{t} p(s)\left(h_{2}\left(\delta(s), t_{0}\right)\right)^{r} \Delta s \leq 0
$$

which yields that

$$
r\left(t_{5}\right) z^{\Delta \Delta}\left(t_{5}\right) \geq(P k)^{\gamma} \int_{t_{5}}^{t} p(s)\left(h_{2}\left(\delta(s), t_{0}\right)\right)^{r} \Delta s
$$

which contradicts (2.26). Hence $Z(t)>0$ and $z^{\Delta}(t) / t$ is nonincreasing. The proof is complete.

Lemma 2.4. Assume that $(H)$ holds and $x$ is a solution of (1.2) which satisfies case (iii) of Lemma 2.1. If

$$
\int_{t_{0}}^{\infty} p(s) R^{\sigma}(s) \Delta s=\infty
$$

where $R(t):=\int_{t_{0}}^{t}(\sigma(u) / r(u)) \Delta u$ for $t \in\left[t_{0}, \infty\right)_{\mathbb{T}}$, then $\lim _{t \rightarrow \infty} x(t)=0$.

Proof. Let $x$ be a solution of (1.2) such that case (iii) of Lemma 2.1 holds for $t \geq t_{1}$. Then $\lim _{t \rightarrow \infty} z(t)=l \geq 0, \lim _{t \rightarrow \infty} x(t)=l /(1-a) \geq 0$. Next we claim that $l=0$. Otherwise, there exists $t_{2} \geq t_{1}$ such that $z(\delta(t)) \geq l>0$ for all $t \geq t_{2}$. By the definition of $z$, we have that (2.35) holds. Integrating both sides of (2.35) from $t$ to $\infty$, we get

$$
z^{\Delta \Delta}(t) \geq \frac{1}{r(t)} \int_{t}^{\infty} p(s) z^{\gamma}(\delta(s)) \Delta s
$$

Integrating again from $t$ to $\infty$, we have

$$
-z^{\Delta}(t) \geq \int_{t}^{\infty} \frac{1}{r(u)} \int_{u}^{\infty} p(s) z^{\gamma}(\delta(s)) \Delta s \Delta u
$$


Integrating again from $t_{2}$ to $\infty$, we obtain

$$
z\left(t_{1}\right) \geq \int_{t_{2}}^{\infty} \int_{v}^{\infty} \frac{1}{r(u)} \int_{u}^{\infty} p(s) z^{r}(\delta(s)) \Delta s \Delta u \Delta v \geq l^{\gamma} \int_{t_{2}}^{\infty} \int_{v}^{\infty} \frac{1}{r(u)} \int_{u}^{\infty} p(s) \Delta s \Delta u \Delta v,
$$

which contradicts (2.38), since by [23, Lemma 1] and [3, Remark 4.7], we get

$$
\begin{aligned}
\int_{t_{0}}^{\infty} \int_{v}^{\infty} & \frac{1}{r(u)} \int_{u}^{\infty} p(s) \Delta s \Delta u \Delta v \\
\quad & \int_{t_{0}}^{\infty} \int_{v}^{\infty} \int_{u}^{\infty} \frac{1}{r(u)} p(s) \Delta s \Delta u \Delta v \\
& =\int_{t_{0}}^{\infty} \int_{v}^{\infty} \int_{v}^{\sigma(s)} \frac{1}{r(u)} p(s) \Delta u \Delta s \Delta v=\int_{t_{0}}^{\infty} \int_{t_{0}}^{\sigma(s)} \int_{v}^{\sigma(s)} \frac{1}{r(u)} p(s) \Delta u \Delta v \Delta s \\
& =\int_{t_{0}}^{\infty} p(s) \int_{t_{0}}^{\sigma(s)} \int_{v}^{\sigma(s)} \frac{1}{r(u)} \Delta u \Delta v \Delta s=\int_{t_{0}}^{\infty} p(s) \int_{t_{0}}^{\sigma(s)} \int_{t_{0}}^{\sigma(u)} \frac{1}{r(u)} \Delta v \Delta u \Delta s \\
& =\int_{t_{0}}^{\infty} p(s) \int_{t_{0}}^{\sigma(s)} \frac{1}{r(u)} \int_{t_{0}}^{\sigma(u)} \Delta v \Delta u \Delta s \\
& =\int_{t_{0}}^{\infty} p(s) \int_{t_{0}}^{\sigma(s)} \frac{\sigma(u)-t_{0}}{r(u)} \Delta u \Delta s=\int_{t_{0}}^{\infty} p(s) \int_{t_{0}}^{\sigma(s)} \frac{\sigma(u)}{r(u)} \Delta u \Delta s=\int_{t_{0}}^{\infty} p(s) R^{\sigma}(s) \Delta s .
\end{aligned}
$$

Hence $\lim _{t \rightarrow \infty} x(t)=0$ and completes the proof.

Theorem 2.5. Assume that $(H),(2.2),(2.26)$, and (2.38) hold, $\gamma \geq 1$. Furthermore, assume that there exists a positive function $\eta \in C_{r d}^{1}\left(\left[t_{0}, \infty\right)_{\mathbb{T}}, \mathbb{R}\right)$ such that for some $0<k<1$ and for all constants $M>0$

$$
\limsup _{t \rightarrow \infty} \int_{t_{0}}^{t}\left(\eta(s) p(s) \zeta(s)-\frac{r(s)\left(\eta^{\Delta}(s)\right)^{2}}{4 k \gamma M^{\gamma-1} \eta(s)}\right) \Delta s=\infty
$$

where $\zeta(t):=\left(h_{2}\left(\delta(t), t_{0}\right) / t\right)^{\gamma}$. Then every solution $x$ of (1.2) oscillates or $\lim _{t \rightarrow \infty} x(t)=0$.

Proof. Suppose that (1.2) has a nonoscillatory solution $x$. We may assume without loss of generality that $x(t)>0, x(\tau(t))>0$, and $x(\delta(t))>0$ for all $t \in\left[t_{1}, \infty\right)_{\mathbb{T}}, t_{1} \in\left[t_{0}, \infty\right)_{\mathbb{T}}$. Then by Lemma 2.1, $z$ satisfies three cases. Assume that $z$ satisfies case (i). Define the function $\omega$ by

$$
\omega(t)=\eta(t) \frac{r(t) z^{\Delta \Delta}(t)}{\left(z^{\Delta}(t)\right)^{\gamma}}, \quad t \in\left[t_{1}, \infty\right)_{\mathbb{T}} .
$$

Then $\omega(t)>0$. Using the product rule, we have

$$
\omega^{\Delta}(t)=\left(r(t) z^{\Delta \Delta}(t)\right)^{\sigma}\left[\frac{\eta(t)}{\left(z^{\Delta}(t)\right)^{\gamma}}\right]^{\Delta}+\left(r(t) z^{\Delta \Delta}(t)\right)^{\Delta} \frac{\eta(t)}{\left(z^{\Delta}(t)\right)^{\gamma}} .
$$


By the quotient rule, we get

$$
\omega^{\Delta}(t)=\left(r(t) z^{\Delta \Delta}(t)\right)^{\sigma} \frac{\eta^{\Delta}(t)\left(z^{\Delta}(t)\right)^{\gamma}-\eta(t)\left(\left(z^{\Delta}(t)\right)^{\gamma}\right)^{\Delta}}{\left(z^{\Delta}(t)\right)^{\gamma}\left(z^{\Delta \sigma}(t)\right)^{\gamma}}+\left(r(t) z^{\Delta \Delta}(t)\right)^{\Delta} \frac{\eta(t)}{\left(z^{\Delta}(t)\right)^{\gamma}} .
$$

By the definition of $z$ and (1.2), we obtain (2.35). From (2.35) and (2.44), we have

$$
\omega^{\Delta}(t) \leq \frac{\eta^{\Delta}(t)}{\eta^{\sigma}(t)} \omega^{\sigma}(t)-\eta(t) p(t) \frac{z^{\gamma}(\delta(t))}{\left(z^{\Delta}(t)\right)^{\gamma}}-\eta(t) \frac{\left(r(t) z^{\Delta \Delta}(t)\right)^{\sigma}\left(\left(z^{\Delta}(t)\right)^{\gamma}\right)^{\Delta}}{\left(z^{\Delta}(t)\right)^{\gamma}\left(z^{\Delta \sigma}(t)\right)^{\gamma}},
$$

from (2.25) and (2.27), for any $0<k<1$, we obtain

$$
\frac{z^{\gamma}(\delta(t))}{\left(z^{\Delta}(t)\right)^{\gamma}}=\frac{z^{\gamma}(\delta(t))}{\left(z^{\Delta}(\delta(t))\right)^{\gamma}} \frac{\left(z^{\Delta}(\delta(t))\right)^{\gamma}}{\left(z^{\Delta}(t)\right)^{\gamma}} \geq\left(k^{1 / \gamma} \frac{h_{2}\left(\delta(t), t_{0}\right)}{\delta(t)}\right)^{\gamma}\left(\frac{\delta(t)}{t}\right)^{\gamma}=k\left(\frac{h_{2}\left(\delta(t), t_{0}\right)}{t}\right)^{\gamma}
$$

hence by (2.48), we have

$$
\omega^{\Delta}(t) \leq \frac{\eta^{\Delta}(t)}{\eta^{\sigma}(t)} \omega^{\sigma}(t)-k \eta(t) p(t) \zeta(t)-\eta(t) \frac{\left(r(t) z^{\Delta \Delta}(t)\right)^{\sigma}\left(\left(z^{\Delta}(t)\right)^{\gamma}\right)^{\Delta}}{\left(z^{\Delta}(t)\right)^{\gamma}\left(z^{\Delta \sigma}(t)\right)^{\gamma}} .
$$

In view of $\gamma \geq 1$, from (2.1) and (i) of Lemma 2.1, we have

$$
\begin{aligned}
\left(\left(z^{\Delta}(t)\right)^{\gamma}\right)^{\Delta} & =\gamma \int_{0}^{1}\left[h z^{\Delta \sigma}(t)+(1-h) z^{\Delta}(t)\right]^{\gamma-1} z^{\Delta \Delta}(t) \mathrm{d} h \\
& \geq \gamma\left(z^{\Delta}(t)\right)^{\gamma-1} z^{\Delta \Delta}(t) \geq \gamma M^{\gamma-1} z^{\Delta \Delta}(t),
\end{aligned}
$$

where $M=z^{\Delta}\left(t_{1}\right)$. By (2.49), we have

$$
\omega^{\Delta}(t) \leq \frac{\eta^{\Delta}(t)}{\eta^{\sigma}(t)} \omega^{\sigma}(t)-k \eta(t) p(t) \zeta(t)-\gamma M^{\gamma-1} \eta(t) \frac{\left[\left(r(t) z^{\Delta \Delta}(t)\right)^{\sigma}\right]^{2}}{\left(z^{\Delta}(t)\right)^{\gamma}\left(z^{\Delta \sigma}(t)\right)^{\gamma}} \frac{z^{\Delta \Delta}(t)}{r^{\sigma}(t) z^{\Delta \Delta \sigma}(t)}
$$

from (i), we have $z^{\Delta}(t) \leq z^{\Delta \sigma}(t)$, by $\left(r(t) z^{\Delta \Delta}(t)\right)^{\Delta}<0$, we have

$$
z^{\Delta \Delta}(t) \geq \frac{r^{\sigma}(t)}{r(t)} z^{\Delta \Delta \sigma}(t)
$$


so we get

$$
\omega^{\Delta}(t) \leq \frac{\eta^{\Delta}(t)}{\eta^{\sigma}(t)} \omega^{\sigma}(t)-k \eta(t) p(t) \zeta(t)-\gamma M^{\gamma-1} \eta(t) \frac{\left[\left(r(t) z^{\Delta \Delta}(t)\right)^{\sigma}\right]^{2}}{r(t)\left(\left(z^{\Delta \sigma}(t)\right)^{\gamma}\right)^{2}},
$$

by (2.44), we have

$$
\omega^{\Delta}(t) \leq \frac{\eta^{\Delta}(t)}{\eta^{\sigma}(t)} \omega^{\sigma}(t)-k \eta(t) p(t) \zeta(t)-\gamma M^{\gamma-1} \frac{\eta(t)}{r(t)\left(\eta^{\sigma}(t)\right)^{2}}\left(\omega^{\sigma}(t)\right)^{2}
$$

Therefore, we obtain

$$
\omega^{\Delta}(t) \leq-k \eta(t) p(t) \zeta(t)+\frac{r(t)\left(\eta^{\Delta}(t)\right)^{2}}{4 \gamma M^{r-1} \eta(t)} .
$$

Integrating inequality (2.55) from $t_{1}$ to $t$, we obtain

$$
-\omega\left(t_{1}\right) \leq \omega(t)-\omega\left(t_{1}\right) \leq-\int_{t_{1}}^{t}\left(k \eta(s) p(s) \zeta(s)-\frac{r(s)\left(\eta^{\Delta}(s)\right)^{2}}{4 \gamma M^{\gamma-1} \eta(s)}\right) \Delta s,
$$

which yields

$$
\int_{t_{1}}^{t}\left(k \eta(s) p(s) \zeta(s)-\frac{r(s)\left(\eta^{\Delta}(s)\right)^{2}}{4 \gamma M^{\gamma-1} \eta(s)}\right) \Delta s \leq \omega\left(t_{1}\right)
$$

for all large $t$, which contradicts (2.43). If (ii) holds, from Lemma 2.1, then $\lim _{t \rightarrow \infty} x(t)=0$. If case (iii) holds, by Lemma 2.4 , then $\lim _{t \rightarrow \infty} x(t)=0$. The proof is complete.

Remark 2.6. From Theorem 2.5, we can obtain different conditions for oscillation of all solutions of (1.2) with different choices of $\eta$.

For example, let $\eta(t)=t$. Now Theorem 2.5 yields the following result.

Corollary 2.7. Assume that (H), (2.2), (2.26), and (2.38) hold, $\gamma \geq 1$. If

$$
\limsup _{t \rightarrow \infty} \int_{t_{0}}^{t}\left(s p(s)\left(\frac{h_{2}\left(\delta(s), t_{0}\right)}{s}\right)^{\gamma}-\frac{r(s)}{4 k \gamma M^{\gamma-1} s}\right) \Delta s=\infty
$$

holds for some $0<k<1$ and for all constants $M>0$, then every solution $x$ of (1.2) is either oscillatory or $\lim _{t \rightarrow \infty} x(t)=0$.

For example, let $\eta(t)=1$. From Theorem 2.5, we have the following result which can be considered as the extension of the Leighton-Wintner Theorem. 
Corollary 2.8. Assume that (H), (2.2), (2.26), and (2.38) hold, and $\gamma \geq 1$. If

$$
\limsup _{t \rightarrow \infty} \int_{t_{0}}^{t} p(s)\left(\frac{h_{2}\left(\delta(s), t_{0}\right)}{s}\right)^{\gamma} \Delta s=\infty,
$$

then every solution $x$ of (1.2) is either oscillatory or $\lim _{t \rightarrow \infty} x(t)=0$.

In the following theorem, we present a new Kamenev-type oscillation criteria for (1.2).

Theorem 2.9. Assume that $(H),(2.2),(2.26)$, and (2.38) hold, $\gamma \geq 1$. Let $\zeta$ and $\eta$ be as defined in Theorem 2.5. If for some $0<k<1$ and for all constants $M>0$

$$
\limsup _{t \rightarrow \infty} \frac{1}{t^{m}} \int_{t_{0}}^{t}\left((t-s)^{m} \eta(s) p(s) \zeta(s)-\frac{r(s) B^{2}(t, s)\left(\eta^{\sigma}(s)\right)^{2}}{4 k \gamma M^{\gamma-1} \eta(s)(t-s)^{m}}\right) \Delta s=\infty,
$$

where $m>1$, and

$$
B(t, s)=(t-s)^{m} \frac{\eta^{\Delta}(s)}{\eta^{\sigma}(s)}-m(t-\sigma(s))^{m-1}, \quad t \geq \sigma(s) \geq t_{0},
$$

then every solution $x$ of (1.2) oscillates or $\lim _{t \rightarrow \infty} x(t)=0$.

Proof. Suppose that (1.2) has a nonoscillatory solution $x$. We may assume without loss of generality that $x(t)>0, x(\tau(t))>0$, and $x(\delta(t))>0$ for all $t \in\left[t_{1}, \infty\right)_{\mathbb{T}}, t_{1} \in\left[t_{0}, \infty\right)_{\mathbb{T}}$. Then by Lemma 2.1, $z$ satisfies three cases. Assume that $z$ satisfies case (i). We proceed as in the proof of Theorem 2.5 to get (2.54) for all $t \geq t_{1}$ sufficiently large. Multiplying (2.54) by $(t-s)^{m}$ and integrating from $t_{1}$ to $t$, we have

$$
\begin{aligned}
\int_{t_{1}}^{t}(t-s)^{m} k \eta(s) p(s) \zeta(s) \Delta s \leq & -\int_{t_{1}}^{t}(t-s)^{m} \omega^{\Delta}(s) \Delta s+\int_{t_{1}}^{t}(t-s)^{m} \frac{\eta^{\Delta}(s)}{\eta^{\sigma}(s)} \omega^{\sigma}(s) \Delta s \\
& -\int_{t_{1}}^{t} \frac{(t-s)^{m} \gamma M^{\gamma-1} \eta(s)}{r(s)\left(\eta^{\sigma}(s)\right)^{2}}\left(\omega^{\sigma}(s)\right)^{2} \Delta s .
\end{aligned}
$$

Integration by parts, we obtain

$$
-\int_{t_{1}}^{t}(t-s)^{m} \omega^{\Delta}(s) \Delta s=-\left.(t-s)^{m} \omega(s)\right|_{t_{1}} ^{t}+\int_{t_{1}}^{t}\left((t-s)^{m}\right)^{\Delta_{s}} \omega^{\sigma}(s) \Delta s
$$

Next, we show that if $t \geq \sigma(s)$ and $m \geq 1$, then

$$
\left((t-s)^{m}\right)^{\Delta_{s}} \leq-m(t-\sigma(s))^{m-1} .
$$


If $\mu(s)=0$, it is easy to see that (2.64) is an equality. If $\mu(s)>0$, then we get

$$
\left((t-s)^{m}\right)^{\Delta_{s}}=\frac{1}{\mu(s)}\left[(t-\sigma(s))^{m}-(t-s)^{m}\right]=-\frac{1}{\sigma(s)-s}\left[(t-s)^{m}-(t-\sigma(s))^{m}\right] .
$$

Using the inequality

$$
x^{m}-y^{m} \geq m y^{m-1}(x-y), \quad x \geq y>0, m \geq 1,
$$

we obtain for $t \geq \sigma(s)$

$$
\left[(t-s)^{m}-(t-\sigma(s))^{m}\right] \geq m(t-\sigma(s))^{m-1}(\sigma(s)-s),
$$

and from this we see that (2.64) holds. From (2.62)-(2.64), we get

$$
\begin{aligned}
\int_{t_{1}}^{t}(t-s)^{m} k \eta(s) p(s) \zeta(s) \Delta s \\
\leq\left(t-t_{1}\right)^{m} \omega\left(t_{1}\right)+\int_{t_{1}}^{t}\left[(t-s)^{m} \frac{\eta^{\Delta}(s)}{\eta^{\sigma}(s)}-m(t-\sigma(s))^{m-1}\right] \omega^{\sigma}(s) \Delta s \\
\quad-\int_{t_{1}}^{t} \frac{(t-s)^{m} \gamma M^{r-1} \eta(s)}{r(s)\left(\eta^{\sigma}(s)\right)^{2}}\left(\omega^{\sigma}(s)\right)^{2} \Delta s .
\end{aligned}
$$

Thus

$$
\int_{t_{1}}^{t}\left((t-s)^{m} \eta(s) p(s) \zeta(s)-\frac{r(s) B^{2}(t, s)\left(\eta^{\sigma}(s)\right)^{2}}{4 k \gamma M^{\gamma-1} \eta(s)(t-s)^{m}}\right) \Delta s \leq \frac{1}{k} \omega\left(t_{1}\right)\left(t-t_{1}\right)^{m},
$$

which implies that

$$
\frac{1}{t^{m}} \int_{t_{1}}^{t}\left((t-s)^{m} \eta(s) p(s) \zeta(s)-\frac{r(s) B^{2}(t, s)\left(\eta^{\sigma}(s)\right)^{2}}{4 k \gamma M^{\gamma-1} \eta(s)(t-s)^{m}}\right) \Delta s \leq \frac{1}{k} \omega\left(t_{1}\right)\left(\frac{t-t_{1}}{t}\right)^{m} .
$$

This easily leads to a contradiction of (2.60). If (ii) holds, from Lemma 2.1, then $\lim _{t \rightarrow \infty} x(t)=$ 0 . If (iii) holds, by Lemma 2.4, then $\lim _{t \rightarrow \infty} x(t)=0$. The proof is complete.

In the following theorem, we present a new Philos-type oscillation criteria for (1.2).

Theorem 2.10. Assume that $(H),(2.2),(2.26)$, and (2.38) hold, $\gamma \geq 1$. Let $\zeta$ and $\eta$ be as defined in Theorem 2.5. Furthermore, assume that there exist functions $H, h \in C_{r d}(\mathbb{D}, \mathbb{R})$, where $\mathbb{D} \equiv\{(t, s)$ : $t \geq s \geq t_{0}$ such that

$$
H(t, t)=0, \quad t \geq t_{0}, \quad H(t, s)>0, \quad t>s \geq t_{0},
$$

and $H$ has a nonpositive continuous $\Delta$-partial derivation $H^{\Delta_{s}}(t, s)$ with respect to the second variable and satisfies 


$$
H^{\Delta_{s}}(t, s)+H(t, s) \frac{\eta^{\Delta}(s)}{\eta^{\sigma}(s)}=-\frac{h(t, s)}{\eta^{\sigma}(s)} \sqrt{H(t, s)} .
$$

If for some $0<k<1$ and for all constants $M>0$

$$
\limsup _{t \rightarrow \infty} \frac{1}{H\left(t, t_{0}\right)} \int_{t_{0}}^{t} K(t, s) \Delta s=\infty
$$

where

$$
K(t, s)=H(t, s) \eta(s) p(s) \zeta(s)-\frac{r(s)\left(h_{-}(t, s)\right)^{2}}{4 k \gamma M^{\gamma-1} \eta(s)}
$$

where $h_{-}(t, s)=\max \{0,-h(t, s)\}$, then every solution $x$ of $(1.2)$ oscillates or $\lim _{t \rightarrow \infty} x(t)=0$.

Proof. Suppose that (1.2) has a nonoscillatory solution $x$. We may assume without loss of generality that $x(t)>0, x(\tau(t))>0$, and $x(\delta(t))>0$ for all $t \in\left[t_{1}, \infty\right)_{\mathbb{T}}, t_{1} \in\left[t_{0}, \infty\right)_{\mathbb{T}}$. Then by Lemma 2.1, $z$ satisfies three cases. Assume that $z$ satisfies case (i). We proceed as in the proof of Theorem 2.5 to get (2.54) for all $t \geq t_{1}$ sufficiently large. Multiplying both sides of (2.54), with $t$ replaced by $s$, by $H(t, s)$, integrating with respect to $s$ from $t_{1}$ to $t$, we have

$$
\begin{aligned}
& \int_{t_{1}}^{t} k H(t, s) \eta(s) p(s) \zeta(s) \Delta s \\
& \quad \leq-\int_{t_{1}}^{t} H(t, s) \omega^{\Delta}(s) \Delta s+\int_{t_{1}}^{t} H(t, s) \frac{\eta^{\Delta}(s)}{\eta^{\sigma}(s)} \omega^{\sigma}(s) \Delta s-\int_{t_{1}}^{t} H(t, s) \frac{r M^{\gamma-1} \eta(s)}{r(s)\left(\eta^{\sigma}(s)\right)^{2}}\left(\omega^{\sigma}(s)\right)^{2} \Delta s .
\end{aligned}
$$

Integrating by parts and using (2.71) and (2.72), we obtain

$$
\begin{aligned}
\int_{t_{1}}^{t} k H(t, s) \eta(s) p(s) \zeta(s) \Delta s & \\
\leq & H\left(t, t_{1}\right) \omega\left(t_{1}\right)+\int_{t_{1}}^{t} H^{\Delta_{s}}(t, s) \omega^{\sigma}(s) \Delta s+\int_{t_{1}}^{t} H(t, s) \frac{\eta^{\Delta}(s)}{\eta^{\sigma}(s)} \omega^{\sigma}(s) \Delta s \\
& -\int_{t_{1}}^{t} H(t, s) \frac{\gamma M^{\gamma-1} \eta(s)}{r(s)\left(\eta^{\sigma}(s)\right)^{2}}\left(\omega^{\sigma}(s)\right)^{2} \Delta s \\
\leq & H\left(t, t_{1}\right) \omega\left(t_{1}\right)+\int_{t_{1}}^{t}\left[-\frac{h(t, s)}{\eta^{\sigma}(s)} \sqrt{H(t, s)} \omega^{\sigma}(s)-H(t, s) \frac{\gamma M^{\gamma-1} \eta(s)}{r(s)\left(\eta^{\sigma}(s)\right)^{2}}\left(\omega^{\sigma}(s)\right)^{2}\right] \Delta s \\
\leq & H\left(t, t_{1}\right) \omega\left(t_{1}\right)+\int_{t_{1}}^{t}\left[\frac{h_{-}(t, s)}{\eta^{\sigma}(s)} \sqrt{H(t, s)} \omega^{\sigma}(s)-H(t, s) \frac{\gamma M^{\gamma-1} \eta(s)}{r(s)\left(\eta^{\sigma}(s)\right)^{2}}\left(\omega^{\sigma}(s)\right)^{2}\right] \Delta s \\
\leq & H\left(t, t_{1}\right) \omega\left(t_{1}\right)+\int_{t_{1}}^{t} \frac{r(s)\left(h_{-}(t, s)\right)^{2}}{4 \gamma M^{\gamma-1} \eta(s)} \Delta s .
\end{aligned}
$$


Therefore, we get

$$
\int_{t_{1}}^{t}\left(H(t, s) \eta(s) p(s) \zeta(s)-\frac{r(s)\left(h_{-}(t, s)\right)^{2}}{4 k \gamma M^{\gamma-1} \eta(s)}\right) \Delta s \leq \frac{1}{k} H\left(t, t_{1}\right) \omega\left(t_{1}\right) .
$$

This easily leads to a contradiction of (2.73). If case (ii) holds, from Lemma 2.1, then $\lim _{t \rightarrow \infty} x(t)=0$. If case (iii) holds, by Lemma 2.4, then $\lim _{t \rightarrow \infty} x(t)=0$. The proof is complete.

The following result can be considered as the extension of the Atkinson's theorem [39].

Theorem 2.11. Assume that (H), (2.2), (2.26), and (2.38) hold, $\gamma>1$. If

$$
\limsup _{t \rightarrow \infty} \int_{t_{0}}^{t} \frac{p(s)}{r(s)} \sigma(s)\left(\frac{h_{2}\left(\delta(s), t_{0}\right)}{\sigma(s)}\right)^{r} \Delta s=\infty,
$$

then every solution $x$ of (1.2) is either oscillatory or $\lim _{t \rightarrow \infty} x(t)=0$.

Proof. Suppose that (1.2) has a nonoscillatory solution $x$. We may assume without loss of generality that $x(t)>0, x(\tau(t))>0$ and $x(\delta(t))>0$ for all $t \in\left[t_{1}, \infty\right)_{\mathbb{T}}, t_{1} \in\left[t_{0}, \infty\right)_{\mathbb{T}}$. Then by Lemma 2.1, $z$ satisfies three cases. Assume that $z$ satisfies case (i). Define the function $\omega$

$$
\omega(t)=t \frac{r(t) z^{\Delta \Delta}(t)}{\left(z^{\Delta}(t)\right)^{\gamma}}, \quad t \in\left[t_{1}, \infty\right)_{\mathbb{T}} .
$$

Using the product rule, (2.25) and (2.27), for any $0<k<1$, we have that

$$
\frac{z^{\gamma}(\delta(t))}{\left(z^{\Delta \sigma}(t)\right)^{\gamma}}=\frac{z^{\gamma}(\delta(t))}{\left(z^{\Delta}(\delta(t))\right)^{\gamma}} \frac{\left(z^{\Delta}(\delta(t))\right)^{\gamma}}{\left(z^{\Delta \sigma}(t)\right)^{\gamma}} \geq\left(k^{1 / \gamma} \frac{h_{2}\left(\delta(t), t_{0}\right)}{\delta(t)}\right)^{\gamma}\left(\frac{\delta(t)}{\sigma(t)}\right)^{\gamma}=k\left(\frac{h_{2}\left(\delta(t), t_{0}\right)}{\sigma(t)}\right)^{\gamma} .
$$

By (1.2), we have that (2.35) holds, then from (2.80), we calculate

$$
\begin{aligned}
\omega^{\Delta}(t) & =\left\{r(t) z^{\Delta \Delta}(t)+\sigma(t)\left(r(t) z^{\Delta \Delta}(t)\right)^{\Delta}\right\}\left(z^{\Delta \sigma}(t)\right)^{-\gamma}+\operatorname{tr}(t) z^{\Delta \Delta}(t)\left(\left(z^{\Delta}(t)\right)^{-\gamma}\right)^{\Delta} \\
& \leq r(t) z^{\Delta \Delta}(t)\left(z^{\Delta \sigma}(t)\right)^{-\gamma}-\sigma(t) p(t)\left(\frac{z(\delta(t))}{z^{\Delta \sigma}(t)}\right)^{\gamma}+\operatorname{tr}(t) z^{\Delta \Delta}(t)\left(\left(z^{\Delta}(t)\right)^{-\gamma}\right)^{\Delta} \\
& \leq r(t) \frac{\left(\left(z^{\Delta}(t)\right)^{1-\gamma}\right)^{\Delta}}{1-\gamma}-k \sigma(t) p(t)\left(\frac{h_{2}\left(\delta(t), t_{0}\right)}{\sigma(t)}\right)^{\gamma},
\end{aligned}
$$


where the last inequality is true because $\left(\left(z^{\Delta}(t)\right)^{-\gamma}\right)^{\Delta} \leq 0$ due to (2.1) and because

$$
\begin{aligned}
\left(\left(z^{\Delta}(t)\right)^{1-\gamma}\right)^{\Delta} & =(1-\gamma) \int_{0}^{1}\left[h z^{\Delta \sigma}(t)+(1-h) z^{\Delta}(t)\right]^{-\gamma} z^{\Delta \Delta}(t) \mathrm{d} h \\
& \leq(1-\gamma) \int_{0}^{1}\left[h z^{\Delta \sigma}(t)+(1-h) z^{\Delta \sigma}(t)\right]^{-\gamma} z^{\Delta \Delta}(t) \mathrm{d} h \\
& =(1-\gamma)\left(z^{\Delta \sigma}(t)\right)^{-\gamma} z^{\Delta \Delta}(t) .
\end{aligned}
$$

Upon integration we arrive at

$$
\begin{aligned}
\int_{t_{1}}^{t} k \sigma(s) \frac{p(s)}{r(s)}\left(\frac{h_{2}\left(\delta(s), t_{0}\right)}{\sigma(s)}\right)^{\gamma} \Delta s & \leq \int_{t_{1}}^{t} \frac{\left(\left(z^{\Delta}(s)\right)^{1-\gamma}\right)^{\Delta}}{1-\gamma} \Delta s-\int_{t_{1}}^{t} \frac{\omega^{\Delta}(s)}{r(s)} \Delta s \\
& =\frac{\left(z^{\Delta}(t)\right)^{1-\gamma}}{1-\gamma}-\frac{\left(z^{\Delta}\left(t_{1}\right)\right)^{1-\gamma}}{1-\gamma}-\int_{t_{1}}^{t} \frac{\omega^{\Delta}(s)}{r(s)} \Delta s \\
& \leq \frac{\left(z^{\Delta}\left(t_{1}\right)\right)^{1-\gamma}}{\gamma-1}+\frac{\omega\left(t_{1}\right)}{r\left(t_{1}\right)}-\frac{\omega(t)}{r(t)}+\int_{t_{1}}^{t} \omega^{\sigma}(s)\left(\frac{1}{r(s)}\right)^{\Delta} \Delta s \\
& \leq \frac{\left(z^{\Delta}\left(t_{1}\right)\right)^{1-\gamma}}{\gamma-1}+\frac{\omega\left(t_{1}\right)}{r\left(t_{1}\right)}
\end{aligned}
$$

from $r^{\Delta}(t) \geq 0$. This contradicts (2.78). If case (ii) holds, from Lemma 2.1, then $\lim _{t \rightarrow \infty} x(t)=$ 0 . If case (iii) holds, by Lemma 2.4 , then $\lim _{t \rightarrow \infty} x(t)=0$. The proof is complete.

Theorem 2.12. Assume that $(H),(2.2),(2.26)$, and (2.38) hold, $\gamma \leq 1$. Furthermore, assume that there exists a positive function $\eta \in C_{r d}^{1}\left(\left[t_{0}, \infty\right)_{\mathbb{T}}, \mathbb{R}\right)$ such that for some $0<k<1$ and for all constants $L>0$

$$
\limsup _{t \rightarrow \infty} \int_{t_{0}}^{t}\left(\eta(s) p(s) \zeta(s)-\frac{r(s)\left(\eta^{\Delta}(s)\right)^{2}}{4 k \gamma(L \sigma(s))^{\gamma-1} \eta(s)}\right) \Delta s=\infty
$$

where $\zeta$ is as defined as in Theorem 2.5. Then every solution $x$ of (1.2) is either oscillatory or $\lim _{t \rightarrow \infty} x(t)=0$.

Proof. Suppose that (1.2) has a nonoscillatory solution $x$. We may assume without loss of generality that $x(t)>0, x(\tau(t))>0$ and $x(\delta(t))>0$ for all $t_{1} t \in\left[t_{1}, \infty\right)_{\mathbb{T}}, \in\left[t_{0}, \infty\right)_{\mathbb{T}}$. Then by Lemma 2.1, $z$ satisfies three cases. Assume $z$ satisfies case (i). Define the function $\omega$ as (2.44). 
We proceed as in the proof of Theorem 2.5 and we get (2.49). In view of $\gamma \leq 1$, from (2.1) and (i) of Lemma 2.1, we have

$$
\begin{aligned}
\left(\left(z^{\Delta}(t)\right)^{\gamma}\right)^{\Delta} & =\gamma \int_{0}^{1}\left[h z^{\Delta \sigma}(t)+(1-h) z^{\Delta}(t)\right]^{\gamma-1} z^{\Delta \Delta}(t) \mathrm{d} h \\
& \geq \gamma\left(z^{\Delta \sigma}(t)\right)^{\gamma-1} z^{\Delta \Delta}(t),
\end{aligned}
$$

from (2.27), there exists a constant $L>0$ such that $z^{\Delta}(t) \leq L t$, so

$$
\left(\left(z^{\Delta}(t)\right)^{\gamma}\right)^{\Delta} \geq \gamma(L \sigma(t))^{\gamma-1} z^{\Delta \Delta}(t)
$$

By (2.49), we have

$$
\omega^{\Delta}(t) \leq \frac{\eta^{\Delta}(t)}{\eta^{\sigma}(t)} \omega^{\sigma}(t)-k \eta(t) p(t) \zeta(t)-\gamma(L \sigma(t))^{\gamma-1} \frac{\eta(t)}{r(t)\left(\eta^{\sigma}(t)\right)^{2}}\left(\omega^{\sigma}(t)\right)^{2}
$$

Therefore, we obtain

$$
\omega^{\Delta}(t) \leq-k \eta(t) p(t) \zeta(t)+\frac{r(t)\left(\eta^{\Delta}(t)\right)^{2}}{4 \gamma(L \sigma(t))^{\gamma-1} \eta(t)} .
$$

Integrating inequality (2.88) from $t_{1}$ to $t$, we obtain

$$
-\omega\left(t_{1}\right) \leq \omega(t)-\omega\left(t_{1}\right) \leq-\int_{t_{1}}^{t}\left(k \eta(s) p(s) \zeta(s)-\frac{r(s)\left(\eta^{\Delta}(s)\right)^{2}}{4 \gamma(L \sigma(s))^{\gamma-1} \eta(s)}\right) \Delta s,
$$

which yields

$$
\int_{t_{1}}^{t}\left(k \eta(s) p(s) \zeta(s)-\frac{r(s)\left(\eta^{\Delta}(s)\right)^{2}}{4 \gamma(L \sigma(s))^{\gamma-1} \eta(s)}\right) \Delta s \leq \omega\left(t_{1}\right)
$$

for all large $t$, which contradicts (2.84). If case (ii) holds, from Lemma 2.1, then $\lim _{t \rightarrow \infty} x(t)=$ 0 . If case (iii) holds, by Lemma 2.4 , then $\lim _{t \rightarrow \infty} x(t)=0$. The proof is complete.

Remark 2.13. From Theorem 2.12, we can obtain different conditions for oscillation of all solutions of (1.2) with different choices of $\eta$.

For example, let $\eta(t)=t$. Now Theorem 2.12 yields the following results.

Corollary 2.14. Assume that (H), (2.2), (2.26), and (2.38) hold, $\gamma \leq 1$. If

$$
\limsup _{t \rightarrow \infty} \int_{t_{0}}^{t}\left(s p(s)\left(\frac{h_{2}\left(\delta(s), t_{0}\right)}{s}\right)^{\gamma}-\frac{r(s)}{4 k \gamma(L \sigma(s))^{\gamma-1} s}\right) \Delta s=\infty
$$


holds for some $0<k<1$ and for all constants $L>0$, then every solution $x$ of (1.2) is either oscillatory or $\lim _{t \rightarrow \infty} x(t)=0$.

For example, let $\eta(t)=1$. From Theorem 2.12, we have the following result which can be considered as the extension of the Leighton-Wintner theorem.

Corollary 2.15. Assume that $(H),(2.2),(2.26)$, and (2.38) hold, $\gamma \leq 1$. If (2.59) holds, then every solution $x$ of (1.2) is either oscillatory or $\lim _{t \rightarrow \infty} x(t)=0$.

In the following theorem, we present a new Kamenev-type oscillation criteria for (1.2).

Theorem 2.16. Assume that (H), (2.2), (2.26), and (2.38) hold, $\gamma \leq 1$. Let $\zeta$ and $\eta$ be as defined in Theorem 2.12. If for some $0<k<1$ and for all constants $L>0$

$$
\limsup _{t \rightarrow \infty} \frac{1}{t^{m}} \int_{t_{0}}^{t}\left((t-s)^{m} \eta(s) p(s) \zeta(s)-\frac{r(s) B^{2}(t, s)\left(\eta^{\sigma}(s)\right)^{2}}{4 k \gamma(L \sigma(s))^{\gamma-1} \eta(s)(t-s)^{m}}\right) \Delta s=\infty,
$$

where $m>1$, and

$$
B(t, s)=(t-s)^{m} \frac{\eta^{\Delta}(s)}{\eta^{\sigma}(s)}-m(t-\sigma(s))^{m-1}, \quad t \geq \sigma(s) \geq t_{0},
$$

then every solution $x$ of (1.2) oscillates or $\lim _{t \rightarrow \infty} x(t)=0$. details.

The proof is similar to that of Theorem 2.9 using inequality (2.88), so we omit the

In the following theorem, we present a new Philos-type oscillation criteria for (1.2).

Theorem 2.17. Assume that (H), (2.2), (2.26), and (2.38) hold, $\gamma \leq 1$. Let $\zeta$ and $\eta$ be as defined in Theorem 2.12. Furthermore, assume that there exist functions $H, h \in C_{r d}(\mathbb{D}, \mathbb{R})$, where $\mathbb{D} \equiv\{(t, s)$ : $\left.t \geq s \geq t_{0}\right\}$ such that (2.71) holds, and $H$ has a nonpositive continuous $\Delta$-partial derivation $H^{\Delta_{s}}(t, s)$ with respect to the second variable and satisfies (2.72). If

$$
\limsup _{t \rightarrow \infty} \frac{1}{H\left(t, t_{0}\right)} \int_{t_{0}}^{t} K(t, s) \Delta s=\infty
$$

holds for some $0<k<1$ and for all constants $L>0$, where

$$
K(t, s)=H(t, s) \eta(s) p(s) \zeta(s)-\frac{r(s)\left(h_{-}(t, s)\right)^{2}}{4 k \gamma(L \sigma(s))^{\gamma-1} \eta(s)},
$$

where $h_{-}(t, s)=\max \{0,-h(t, s)\}$. Then every solution $x$ of (1.2) oscillates or $\lim _{t \rightarrow \infty} x(t)=0$.

The proof is similar to that of the proof of Theorem 2.10 using inequality (2.88), so we omit the details. [40].

The following result can be considered as the extension of the Belohorec's theorem 
Theorem 2.18. Assume that $(H),(2.2),(2.26)$, and (2.38) hold $\gamma<1$. If

$$
\limsup _{t \rightarrow \infty} \int_{t_{0}}^{t} \frac{p(s)}{r^{\gamma}(\delta(s))}\left(h_{2}\left(\delta(s), t_{0}\right)\right)^{r} \Delta s=\infty
$$

then every solution $x$ of (1.2) is either oscillatory or satisfies $\lim _{t \rightarrow \infty} x(t)=0$.

Proof. Suppose that (1.2) has a nonoscillatory solution $x$. We may assume without loss of generality that $x(t)>0, x(\tau(t))>0$, and $x(\delta(t))>0$ for all $t_{1} t \in\left[t_{1}, \infty\right)_{\mathbb{T}}, \in\left[t_{0}, \infty\right)_{\mathbb{T}}$. Then by Lemma 2.1, $z$ satisfies three cases. Assume that $z$ satisfies case (i). From (i) and (2.1) we have

$$
\begin{aligned}
\left(\left(r(t) z^{\Delta \Delta}(t)\right)^{1-\gamma}\right)^{\Delta} & =(1-\gamma) \int_{0}^{1}\left[h\left(r(t) z^{\Delta \Delta}(t)\right)^{\sigma}+(1-h) r(t) z^{\Delta \Delta}(t)\right]^{-\gamma}\left(r(t) z^{\Delta \Delta}(t)\right)^{\Delta} \mathrm{d} h \\
& \leq(1-\gamma) \int_{0}^{1}\left[h r(t) z^{\Delta \Delta}(t)+(1-h) r(t) z^{\Delta \Delta}(t)\right]^{-\gamma}\left(r(t) z^{\Delta \Delta}(t)\right)^{\Delta} \mathrm{d} h \\
& =(1-\gamma)\left(r(t) z^{\Delta \Delta}(t)\right)^{-\gamma}\left(r(t) z^{\Delta \Delta}(t)\right)^{\Delta},
\end{aligned}
$$

so

$$
\left(r(t) z^{\Delta \Delta}(t)\right)^{-\gamma}\left(r(t) z^{\Delta \Delta}(t)\right)^{\Delta} \geq \frac{\left(\left(r(t) z^{\Delta \Delta}(t)\right)^{1-\gamma}\right)^{\Delta}}{1-\gamma} .
$$

By (1.2), we have that (2.35) holds. Using (2.25) and (2.27), for any $0<k<1$, we obtain after dividing (2.35) by $\left(r(t) z^{\Delta \Delta}(t)\right)^{\gamma}$ for all large $t$

$$
\begin{aligned}
0 & \geq \frac{\left(r(t) z^{\Delta \Delta}(t)\right)^{\Delta}+p(t) z^{\gamma}(\delta(t))}{\left(r(t) z^{\Delta \Delta}(t)\right)^{\gamma}} \\
& =\left(r(t) z^{\Delta \Delta}(t)\right)^{-\gamma}\left(r(t) z^{\Delta \Delta}(t)\right)^{\Delta}+p(t)\left(\frac{z(\delta(t))}{r(t) z^{\Delta \Delta}(t)}\right)^{\gamma} \\
& \geq \frac{\left(\left(r(t) z^{\Delta \Delta}(t)\right)^{1-\gamma}\right)^{\Delta}}{1-\gamma}+\frac{p(t)}{r^{\gamma}(\delta(t))}\left(\frac{z(\delta(t))}{z^{\Delta}(\delta(t))} \frac{z^{\Delta}(\delta(t))}{z^{\Delta \Delta}(\delta(t))}\right)^{\gamma} \\
& \geq \frac{\left(\left(r(t) z^{\Delta \Delta}(t)\right)^{1-\gamma}\right)^{\Delta}}{1-\gamma}+\frac{p(t)}{r^{\gamma}(\delta(t))}\left(k \frac{h_{2}\left(\delta(t), t_{0}\right)}{\delta(t)} \delta(t)\right)^{\gamma} \\
& =\frac{\left(\left(r(t) z^{\Delta \Delta}(t)\right)^{1-\gamma}\right)^{\Delta}}{1-\gamma}+k^{\gamma} \frac{p(t)}{r^{\gamma}(\delta(t))}\left(h_{2}\left(\delta(t), t_{0}\right)\right)^{\gamma} .
\end{aligned}
$$


So,

$$
k^{\gamma} \frac{p(t)}{r^{\gamma}(\delta(t))}\left(h_{2}\left(\delta(t), t_{0}\right)\right)^{\gamma} \leq \frac{\left(\left(r(t) z^{\Delta \Delta}(t)\right)^{1-\gamma}\right)^{\Delta}}{\gamma-1} .
$$

Upon integration we arrive at

$$
\int_{t_{1}}^{t} k^{\gamma} \frac{p(s)}{r \gamma(\delta(s))}\left(h_{2}\left(\delta(s), t_{0}\right)\right)^{\gamma} \Delta s \leq \int_{t_{1}}^{t} \frac{\left(\left(r(t) z^{\Delta \Delta}(s)\right)^{1-\gamma}\right)^{\Delta}}{\gamma-1} \Delta s \leq \frac{\left(r\left(t_{1}\right) z^{\Delta \Delta}\left(t_{1}\right)\right)^{1-\gamma}}{1-\gamma} .
$$

This contradicts (2.96). If case (ii) holds, from Lemma 2.1, then $\lim _{t \rightarrow \infty} x(t)=0$. If case (iii) holds, by Lemma 2.4, then $\lim _{t \rightarrow \infty} x(t)=0$. The proof is complete.

Remark 2.19. One can easily see that the results obtained in [14-16, 21, 23, 24, 35] cannot be applied in (1.2), so our results are new.

\section{Examples}

In this section we give the following examples to illustrate our main results.

Example 3.1. Consider the third-order neutral delay dynamic equations on time-scales

$$
\left(x(t)-\frac{1}{2} x(\tau(t))\right)^{\Delta \Delta \Delta}+\frac{\beta}{t}\left(\frac{t}{h_{2}\left(\delta(t), t_{0}\right)}\right)^{\gamma} x^{\gamma}(\delta(t))=0, \quad t \in\left[t_{0}, \infty\right)_{\mathbb{T}},
$$

where $\beta>0,1<\gamma<2$ is a quotient of odd positive integers, $h_{2}\left(\delta(t), t_{0}\right) \leq t^{2}$.

Let $r(t)=1, a(t)=1 / 2, p(t)=(\beta / t)\left(t / h_{2}\left(\delta(t), t_{0}\right)\right)^{\gamma}$. It is easy to see that (2.2), (2.26), and (2.38) hold. Also

$$
\limsup _{t \rightarrow \infty} \int_{t_{0}}^{t} p(s)\left(\frac{h_{2}\left(\delta(s), t_{0}\right)}{s}\right)^{\gamma} \Delta s=\beta \limsup \int_{t \rightarrow \infty}^{t} \frac{\Delta s}{s}=\infty
$$

Hence by Corollary 2.8, every solution $x$ of (3.1) is either oscillatory or $\lim _{t \rightarrow \infty} x(t)=0$.

Example 3.2. Consider the third-order neutral delay differential equation

$$
\left(x(t)-\frac{1}{10} x(t-2)\right)^{\prime \prime \prime}+\left(1-\frac{e^{2}}{10}\right) e^{2 t} x^{3}(t)=0, \quad t \in\left[t_{0}, \infty\right) .
$$

Let $\gamma=3, r(t)=1, a(t)=1 / 10, p(t)=\left(1-e^{2} / 10\right) e^{2 t}$. It is easy to see that all the conditions of Corollary 2.8 hold. Then by Corollary 2.8, every solution $x$ of (3.3) is either oscillatory or satisfies $\lim _{t \rightarrow \infty} x(t)=0$. In fact, $x(t)=e^{-t}$ is a solution of (3.3). 
Example 3.3. Consider the third-order delay dynamic equation

$$
\left(t\left(x(t)-\frac{1}{2} x(\tau(t))\right)^{\Delta \Delta}\right)^{\Delta}+\frac{\beta t^{\gamma-1}}{\delta^{2}(t)} x^{\gamma}(\delta(t))=0, \quad t \in[1, \infty)_{\mathbb{T}}
$$

where $\mathbb{T}=q^{\mathbb{N}_{0}}, \beta>0, \gamma>1$ is a quotient of odd positive integers.

For $\mathbb{T}=q^{\mathbb{N}_{0}}$, we have $h_{2}\left(\delta(t), t_{0}\right)=h_{2}(\delta(t), 1)=(\delta(t)-1)(\delta(t)-q) /(1+q), \sigma(t)=q t$. Let $r(t)=t, p(t)=\beta t^{\gamma-1} / \delta^{2}(t)$. It is easy to see that (2.2) and (2.38) hold, and

$$
\begin{aligned}
\int_{t_{0}}^{\infty} p(t)\left(h_{2}\left(\delta(t), t_{0}\right)\right)^{\gamma} \Delta t & =\beta \int_{1}^{\infty} \frac{t^{\gamma-1}}{\delta^{2}(t)}\left(\frac{(\delta(t)-1)(\delta(t)-q)}{1+q}\right)^{\gamma} \Delta t \\
& \geq l \beta \int_{1}^{\infty} t^{\gamma-1} \Delta t=\infty, \quad \text { for some } 0<l<1 .
\end{aligned}
$$

Hence (2.26) holds. Also

$$
\begin{aligned}
\limsup _{t \rightarrow \infty} \int_{t_{0}}^{t} \frac{p(s)}{r(s)} \sigma(s)\left(\frac{h_{2}\left(\delta(s), t_{0}\right)}{\sigma(s)}\right)^{\gamma} \Delta s \\
\quad=\beta q^{1-\gamma} \limsup _{t \rightarrow \infty} \int_{1}^{t} \frac{1}{s \delta^{2}(s)}\left(\frac{(\delta(s)-1)(\delta(s)-q)}{1+q}\right)^{\gamma} \Delta s=\infty,
\end{aligned}
$$

so (2.78) holds. By Theorem 2.11, every solution $x$ of (3.4) is either oscillatory or satisfies $\lim _{t \rightarrow \infty} x(t)=0$.

Example 3.4. Consider the third-order delay dynamic equation

$$
\left(x(t)-\frac{1}{3} x(\tau(t))\right)^{\Delta \Delta \Delta}+\frac{\beta}{t}\left(\frac{1}{h_{2}\left(\delta(t), t_{0}\right)}\right)^{\gamma} x^{\gamma}(\delta(t))=0, \quad t \in\left[t_{0}, \infty\right)_{\mathbb{T}},
$$

where $h_{2}\left(\delta(t), t_{0}\right) \leq t^{2}, \beta>0, \gamma<1$ is a quotient of odd positive integers.

Let $r(t)=1, p(t)=(\beta / t)\left(1 / h_{2}\left(\delta(t), t_{0}\right)\right)^{\gamma}$. It is easy to see that $(2.2),(2.26)$, and (2.38) hold. Also we have

$$
\limsup _{t \rightarrow \infty} \int_{t_{0}}^{t} \frac{p(s)}{r^{r}(\delta(s))}\left(h_{2}\left(\delta(s), t_{0}\right)\right)^{r} \Delta s=\beta \limsup _{t \rightarrow \infty} \int_{t_{0}}^{t} \frac{\Delta s}{s}=\infty
$$

Hence (2.96) holds. By Theorem 2.18, every solution $x$ of (3.7) is either oscillatory or satisfies $\lim _{t \rightarrow \infty} x(t)=0$. 


\section{Acknowledgments}

The authors sincerely thank the reviewers for their valuable suggestions and useful comments that have to the present improved version of the original manuscript. This research is supported by the Natural Science Foundation of China $(60774004,60904024)$, China Postdoctoral Science Foundation Funded Project (20080441126, 200902564), Shandong Postdoctoral Funded Project (200802018), Shandong Research Funds (Y2008A28), and also supported by University of Jinan Research Funds for Doctors (B0621, XBS0843).

\section{References}

[1] S. Hilger, "Analysis on measure chains-a unified approach to continuous and discrete calculus," Results in Mathematics, vol. 18, no. 1-2, pp. 18-56, 1990.

[2] R. P. Agarwal, M. Bohner, D. O'Regan, and A. Peterson, "Dynamic equations on time scales: a survey," Journal of Computational and Applied Mathematics, vol. 141, no. 1-2, pp. 1-26, 2002.

[3] M. Bohner and G. Sh. Guseinov, "Improper integrals on time scales," Dynamic Systems and Applications, vol. 12, no. 1-2, pp. 45-65, 2003.

[4] M. Bohner and A. Peterson, Dynamic Equations on Time Scales: An Introduction with Application, Birkhäuser, Boston, Mass, USA, 2001.

[5] M. Bohner and A. Peterson, Advances in Dynamic Equations on Time Scales, Birkhäuser, Boston, Mass, USA, 2003.

[6] R. P. Agarwal, M. Bohner, and S. H. Saker, "Oscillation of second order delay dynamic equations," The Canadian Applied Mathematics Quarterly, vol. 13, no. 1, pp. 1-17, 2005.

[7] R. P. Agarwal, D. O’Regan, and S. H. Saker, "Oscillation criteria for second-order nonlinear neutral delay dynamic equations," Journal of Mathematical Analysis and Applications, vol. 300, no. 1, pp. 203 217, 2004.

[8] E. Akin-Bohner and J. Hoffacker, "Oscillation properties of an Emden-Fowler type equation on discrete time scales," Journal of Difference Equations and Applications, vol. 9, no. 6, pp. 603-612, 2003.

[9] E. Akin-Bohner, M. Bohner, and S. H. Saker, "Oscillation criteria for a certain class of second order Emden-Fowler dynamic equations," Electronic Transactions on Numerical Analysis, vol. 27, pp. 1-12, 2007.

[10] M. Bohner and S. H. Saker, "Oscillation of second order nonlinear dynamic equations on time scales," The Rocky Mountain Journal of Mathematics, vol. 34, no. 4, pp. 1239-1254, 2004.

[11] M. Bohner, "Some oscillation criteria for first order delay dynamic equations," Far East Journal of Applied Mathematics, vol. 18, no. 3, pp. 289-304, 2005.

[12] L. Erbe, A. Peterson, and S. H. Saker, "Oscillation criteria for second-order nonlinear dynamic equations on time scales," Journal of the London Mathematical Society, vol. 67, no. 3, pp. 701-714, 2003.

[13] L. Erbe, A. Peterson, and S. H. Saker, "Oscillation criteria for second-order nonlinear delay dynamic equations," Journal of Mathematical Analysis and Applications, vol. 333, no. 1, pp. 505-522, 2007.

[14] L. Erbe, A. Peterson, and S. H. Saker, "Asymptotic behavior of solutions of a third-order nonlinear dynamic equation on time scales," Journal of Computational and Applied Mathematics, vol. 181, no. 1, pp. 92-102, 2005.

[15] L. Erbe, A. Peterson, and S. H. Saker, "Hille and Nehari type criteria for third-order dynamic equations," Journal of Mathematical Analysis and Applications, vol. 329, no. 1, pp. 112-131, 2007.

[16] L. Erbe, A. Peterson, and S. H. Saker, "Oscillation and asymptotic behavior of a third-order nonlinear dynamic equation," The Canadian Applied Mathematics Quarterly, vol. 14, no. 2, pp. 129-147, 2006.

[17] S. R. Grace, R. P. Agarwal, M. Bohner, and D. O’Regan, “Oscillation of second-order strongly superlinear and strongly sublinear dynamic equations," Communications in Nonlinear Science and Numerical Simulation, vol. 14, no. 8, pp. 3463-3471, 2009.

[18] Z. Han, S. Sun, and B. Shi, "Oscillation criteria for a class of second-order Emden-Fowler delay dynamic equations on time scales," Journal of Mathematical Analysis and Applications, vol. 334, no. 2, pp. 847-858, 2007.

[19] Z. Han, T. Li, S. Sun, and C. Zhang, "Oscillation for second-order nonlinear delay dynamic equations on time scales," Advances in Difference Equations, vol. 2009, Article ID 756171, 13 pages, 2009.

[20] T. S. Hassan, "Oscillation criteria for half-linear dynamic equations on time scales," Journal of Mathematical Analysis and Applications, vol. 345, no. 1, pp. 176-185, 2008. 
[21] T. S. Hassan, "Oscillation of third order nonlinear delay dynamic equations on time scales," Mathematical and Computer Modelling, vol. 49, no. 7-8, pp. 1573-1586, 2009.

[22] B. Jia, L. Erbe, and A. Peterson, "New comparison and oscillation theorems for second-order halflinear dynamic equations on time scales," Computers $\mathcal{E}$ Mathematics with Applications, vol. 56, no. 10, pp. 2744-2756, 2008.

[23] B. Karpuz, "Unbounded oscillation of higher-order nonlinear delay dynamic equations of neutral type with oscillating coefficients," Electronic Journal of Qualitative Theory of Differential Equations, vol. 2009, no. 34, pp. 1-14, 2009.

[24] B. Karpuz, "Asymptotic behaviour of bounded solutions of a class of higher-order neutral dynamic equations," Applied Mathematics and Computation, vol. 215, no. 6, pp. 2174-2183, 2009.

[25] T. Li, Z. Han, S. Sun, and D. Yang, "Existence of nonoscillatory solutions to second-order neutral delay dynamic equations on time scales," Advances in Difference Equations, vol. 2009, Article ID 562329, 10 pages, 2009.

[26] R. M. Mathsen, Q.-R. Wang, and H.-W. Wu, “Oscillation for neutral dynamic functional equations on time scales," Journal of Difference Equations and Applications, vol. 10, no. 7, pp. 651-659, 2004.

[27] Y. Şahiner, "Oscillation of second-order delay differential equations on time scales," Nonlinear Analysis: Theory, Methods \& Applications, vol. 63, no. 5-7, pp. e1073-e1080, 2005.

[28] Y. Şahíner, "Oscillation of second-order neutral delay and mixed-type dynamic equations on time scales," Advances in Difference Equations, vol. 2006, Article ID 65626, 9 pages, 2006.

[29] S. H. Saker, "Oscillation criteria of second-order half-linear dynamic equations on time scales," Journal of Computational and Applied Mathematics, vol. 177, no. 2, pp. 375-387, 2005.

[30] S. H. Saker, "Oscillation of nonlinear dynamic equations on time scales," Applied Mathematics and Computation, vol. 148, no. 1, pp. 81-91, 2004.

[31] S. H. Saker, "Oscillation of second-order nonlinear neutral delay dynamic equations on time scales," Journal of Computational and Applied Mathematics, vol. 187, no. 2, pp. 123-141, 2006.

[32] S. H. Saker, "Oscillation of second-order neutral delay dynamic equations of Emden-Fowler type," Dynamic Systems and Applications, vol. 15, no. 3-4, pp. 629-644, 2006.

[33] S. H. Saker, R. P. Agarwal, and D. O’Regan, “Oscillation results for second-order nonlinear neutral delay dynamic equations on time scales," Applicable Analysis, vol. 86, no. 1, pp. 1-17, 2007.

[34] H.-W. Wu, R.-K. Zhuang, and R. M. Mathsen, "Oscillation criteria for second-order nonlinear neutral variable delay dynamic equations," Applied Mathematics and Computation, vol. 178, no. 2, pp. 321-331, 2006.

[35] Z.-H. Yu and Q.-R. Wang, "Asymptotic behavior of solutions of third-order nonlinear dynamic equations on time scales," Journal of Computational and Applied Mathematics, vol. 225, no. 2, pp. 531-540, 2009.

[36] B. G. Zhang and Z. Shanliang, "Oscillation of second-order nonlinear delay dynamic equations on time scales," Computers \& Mathematics with Applications, vol. 49, no. 4, pp. 599-609, 2005.

[37] B. G. Zhang and X. Deng, "Oscillation of delay differential equations on time scales," Mathematical and Computer Modelling, vol. 36, no. 11-13, pp. 1307-1318, 2002.

[38] Z.-Q. Zhu and Q.-R. Wang, "Existence of nonoscillatory solutions to neutral dynamic equations on time scales," Journal of Mathematical Analysis and Applications, vol. 335, no. 2, pp. 751-762, 2007.

[39] F. V. Atkinson, “On second-order non-linear oscillations," Pacific Journal of Mathematics, vol. 5, pp. 643-647, 1955.

[40] Š. Belohorec, "Non-linear oscillations of a certain non-linear second-order differential equation," Matematicko-Fyzikalny Časopis: Slovenskej Akademie Vied, vol. 12, pp. 253-262, 1962. 